\title{
Association of Prenylated Proteins with the Plasma Membrane and the Inner Nuclear Membrane Is Mediated by the Same Membrane-targeting Motifs
}

\author{
Helmut Hofemeister, ${ }^{*}$ Klaus Weber, ${ }^{*}$ and Reimer Stick ${ }^{\dagger \ddagger}$
}

\author{
*Max Planck Institute for Biophysical Chemistry, Department of Biochemistry, D-37018 Göttingen, \\ Germany; and ${ }^{\dagger}$ German Cancer Research Center, Division of Cell Biology, D-69120 \\ Heidelberg, Germany
}

Submitted May 10, 2000; Revised XXXX X, 2000; Accepted July 11, 2000

Monitoring Editor: Joseph Gall

\begin{abstract}
Targeting of nuclear lamins to the inner nuclear envelope membrane requires a nuclear localization signal and CaaX motif-dependent posttranslational modifications, including isoprenylation and carboxyl methylation. These modifications, although necessary for membrane targeting, are not sufficient to mediate stable association with membranes. We show that two variants of lamin B3 (i.e., B3a and B3b) exist in Xenopus oocytes. They are encoded by two alternatively spliced, developmentally regulated mRNAs. The two lamin variants differ greatly in their membrane association in meiotically matured eggs. The presence of an extra cysteine residue (as a potential palmitoylation site) and a basic cluster in conjunction with the CaaX motif function as secondary targeting signals responsible for the stable membrane association of lamin B3b in Xenopus eggs. Moreover, transfection experiments with Green Fluorescent Protein lamin tail chimeras and with a Green Fluorescent Protein N-Ras chimera show that these secondary motifs are sufficient to target proteins to the inner nuclear membrane and/or the plasma membrane. Implications for the intracellular trafficking of doubly lipidated proteins are discussed.
\end{abstract}

\section{INTRODUCTION}

Membrane targeting of certain eukaryotic proteins depends on posttranslational lipidation. A major class of these proteins contains CaaX motifs at their $\mathrm{COOH}$ termini. The CaaX motif is the target of a series of posttranslational modifications, including isoprenylation, proteolytic trimming, and carboxyl methylation. In a first step, a farnesyl or geranylgeranyl lipid is added via a stable thioether linkage to the CaaX cysteine (Glomset and Farnsworth, 1994; Zhang and Casey, 1996). The substrate specificity for farnesyltransferase versus geranylgeranyltransferase is determined by the residue in the $X$ position of the CaaX motif (Casey and Seabra, 1996). Next, a specific protease cleaves the aaX residues from the prenylated protein. Finally, the terminal prenylcysteine is recognized by a prenylcysteine carboxyl methyltransferase (pcCMT) that methylesterifies the carboxyl group. Thus, a hydrophobic $\mathrm{COOH}$-terminal region is generated in an otherwise hydrophilic molecule. Proteins that contain a CaaX motif include the Ras proteins and many other small G proteins, the heterotrimeric large $G$ proteins, fungal mating

\footnotetext{
‡ Corresponding author. E-mail address: r.stick@dkfz.de. Abbreviations used: GFP, Green Fluorescent Protein; NE, nuclear envelope; NLS, nuclear localization signal; pcCMT, prenylcysteine carboxyl methyltransferase; PM, plasma membrane.
}

pheromones, and most nuclear lamins (Glomset and Farnsworth, 1994; Reuther and Der, 2000). Prenylated proteins exert a variety of different functions. For many of these proteins, the importance of prenylation for their function has been shown (Loewinger and McKeon, 1988; Hancock et al., 1989, 1991a; Holtz et al., 1989; Krohne et al., 1989; Kitten and Nigg, 1991; Nigg et al., 1992; Hennekes and Nigg, 1994). Large G proteins are geranylgeranylated, whereas Ras proteins, the fungal mating pheromones, and lamins are farnesylated (Moores et al., 1991; Zhang and Casey, 1996). Proteins carrying the same CaaX-dependent modifications are located in different membrane compartments, indicating that additional factors must be responsible for the specific targeting of these proteins. Targeting of lamins to the inner nuclear membrane depends on isoprenylation and the presence of a nuclear localization signal (NLS) (Loewinger and McKeon, 1988; Holtz et al., 1989; Krohne et al., 1989; Kitten and Nigg, 1991).

CaaX-dependent isoprenylation has been used to explain the different fates of vertebrate A- and B-type lamins during cell division. A-type lamins, which lose their isoprene moiety soon after incorporation into the lamina, become freely soluble upon mitotic nuclear envelope (NE) breakdown (Weber et al., 1989; Beck et al., 1990; Kilic et al., 1999). Somatic B-type lamins, in contrast, are permanently isoprenylated and, although depolymerized during mitosis, remain asso- 
ciated with remnants of NE membranes (Gerace and Blobel, 1980; Stick et al., 1988; Nigg et al., 1992; Hennekes and Nigg, 1994). However, as shown by studies of amphibian egg maturation, the CaaX-dependent modifications are not sufficient to mediate the stable membrane association of lamins. Only a small fraction of lamin B3, the major B-type lamin of Xenopus oocytes and eggs, remains associated with membranes after NE breakdown in the meiotic metaphase. The majority of B3 becomes soluble, although it retains its isoprene moiety and remains carboxyl methylated (FirmbachKraft and Stick, 1993).

Posttranslational modification and membrane targeting of Ras proteins has been studied in great detail (Hancock et al., 1989, 1990, 1991a,b; Jackson et al., 1994; Willumsen et al., 1996; Yokoe and Meyer, 1996; Choy et al., 1999). Prenylated H-Ras and N-Ras undergo a second lipidation that comprises palmitoylation of cysteine residues within the hypervariable region of these proteins in the vicinity of the CaaX motif (Hancock et al., 1989). Prenylated K-Ras, which is not palmitoylated, contains a cluster of six basic amino acid residues within the hypervariable domain (Hancock et al., 1990). Palmitoylation or a basic cluster, in combination with the CaaX-dependent modifications, targets proteins to the plasma membrane (PM). The concept of a two-signal mechanism for subcellular localization seems to be more general. It applies also to proteins that are $\mathrm{NH}_{2}$-terminally myristoylated, many of which also have nearby S-acyl groups or basic clusters (Resh, 1996).

The modifications of CaaX proteins follow a general pathway. Whereas prenyltransferases are soluble (Casey and Seabra, 1996), the enzymes that act in the subsequent processing steps, including the prenyl-CaaX protease, the pc$\mathrm{CMT}$, and the protein palmitoyltransferase, are membrane bound. The prenyl-CaaX protease and the pcCMT are predicted polytopic membrane proteins localized in the endoplasmic reticulum (ER) (Choy et al., 1999; Magee and Marshall, 1999). Significantly, the pcCMT has been shown to be completely excluded from the PM (Dai et al., 1998). A detailed study on Ras proteins (Choy et al., 1999) has demonstrated that the CaaX motif alone targets proteins to the endomembrane system, where they are proteolytically processed and methylated. Further trafficking of Ras to the PM is dependent on either a basic cluster or palmitoylation. Furthermore, palmitoylated Ras is found in the Golgi and peri-Golgi vesicles before PM expression (Choy et al., 1999).

We have determined the expression of two variants of Xenopus lamin B3 in early development by reverse transcription (RT)-PCR and have characterized their membrane association in oocytes and eggs. The two variants are generated by alternative RNA splicing (Döring and Stick, 1990). They differ in their last CaaX-containing exon of 12 amino acid residues. Lamin $\mathrm{B} 3 \mathrm{~b}$ contains both a basic cluster of six lysine and arginine residues and an additional cysteine residue directly adjacent to the CaaX motif, whereas lamin B3a contains none of these motifs in addition to the CaaX tetrapeptide. We show that the two variants differ greatly in membrane association after meiotic NE breakdown. Using site-directed mutagenesis, we show that the presence of an extra cysteine residue, as a potential palmitoylation site, and the basic cluster, in conjunction with the CaaX motif, are responsible for the stable membrane association of lamin B3 in Xenopus eggs. Furthermore, transfection experiments with
Green Fluorescent Protein (GFP) chimera demonstrate that CaaX proteins, carrying either of the secondary membranetargeting signals or both signals, can be targeted to both the $\mathrm{PM}$ and the inner NE membrane.

\section{MATERIALS AND METHODS}

\section{Isolation and Manipulation of Oocytes and Eggs}

Xenopus laevis oocyte and embryo techniques were as described (Firmbach-Kraft and Stick, 1993, 1995). Twenty to $30 \mathrm{nl}$ of the appropriate RNA in water was injected per oocyte. Oocytes were kept at $18^{\circ} \mathrm{C}$.

\section{Plasmid Construction}

The coding region of Xenopus lamin B3a (Stick, 1988) (GenBank accession number X13169), excluding the start methionine codon, was amplified by PCR with the use of a sense primer that contained an XbaI recognition site (5'CCGTCTAGAAGCCACATCTACCCCCAGC3') and an antisense primer that contained an SnaBI recognition site (5'CGCTACGTATTACATGATGGAACAGCTGG3') (recognition sites underlined). Cycling parameters were initial denaturation (3 min, $\left.94^{\circ} \mathrm{C}\right)$, followed by 35 cycles $\left(1 \mathrm{~min}, 94^{\circ} \mathrm{C} ; 1 \mathrm{~min}, 58^{\circ} \mathrm{C} ; 2 \mathrm{~min}, 72^{\circ} \mathrm{C}\right)$, and a final polymerization step $\left(10 \mathrm{~min}, 72^{\circ} \mathrm{C}\right)$. The PCR product was double digested with $\mathrm{XbaI}-\mathrm{SnaBI}$ and cloned into XbaI-SnaBI doubledigested pCS2+(Flag) vector (Rupp et al., 1994). The resulting protein contains at its $\mathrm{NH}_{2}$ terminus the Flag epitope followed by nine vectorderived amino acid residues (MDYKDDDDKNSRPLEPLE) (Flag tag underlined). Flag-B3b was generated by replacing the NheI-NotI fragment of pCS2+Flag-B3a with the corresponding fragment of pCS2+myc-B3b, which contains the lamin B3b-specific COOH-terminal region (Döring and Stick, 1990).

Mutants of lamin B3 were generated by site-directed mutagenesis with the use of the QuickChange mutagenesis kit (Stratagene, La Jolla, CA) with pairs of complementary mutagenic primers. The following primers were used (sense primers only are listed): B3aCC, 5'CCAATCAGTGGACCCCTGCTGTTCCATCATGTAATGG3'; B3b3Q, 5'GTTCCGTTCCCAGACACAACAACAAAAAAAGAAATGTTGTTCAG3'; B3b6Q，5'CCAGACACAACAACAACAACAGCAATGTTGTTCAG3'; B3bSC, 5'GAAGAAAAAAGAAAAGTTGTTCAGTTTCATAATGG3'; B3bCS, 5'GAAGAAAAAAGAAATGTTCTTCAGTTTCATAATGG3'; B3aCSIL, 5'GCTGTTCCATCCTGTAATACG3'; and B3aCAIL, 5'CCAGCTGTGCCATCCTGTAATACG3'. For generation of the B3aCAIL mutant, plasmid pCS2+B3aCSIL was used as a template.

GFP-lamin B3 tail chimeras were generated by amplifying amino acid residues $388-583$ of lamin B3a and B3b with a sense primer that contained an EcoRI recognition site (5'GAGGGAATTCCTGTCACCAAGCCCTTC3') and antisense primers with the $K p n \mathrm{I}$ recognition site 5' GTCTGGTACCACGTATTACATGATGGAACAGC3' for B3a or 5'CTCACGGTACCTCTAGACCATTATGAAACTGAAC3' for $\mathrm{B} 3 \mathrm{~b}$. The PCR products were double digested with EcoRI-KpnI and cloned into the EcoRI-KpnI sites of the eukaryotic expression vector peGFP-C2 (Clontech, Palo Alto, CA) (GFP-B3 constructs). The NLS of lamin B3, KKRKL (amino acid residues 413-417) (Stick, 1988), was inactivated by changing it to TKIKL with a mutagenic primer pair (sense primer, 5'GCCTTTTACGTGGGACAAAGATAAAGTTAGACGAAACTGG3') (GFP-B3ANLS constructs).

To generate the GFP-N-Ras chimera, the coding region of eGFP, excluding the start methionine codon, was amplified by PCR with the use of plasmid peGFP-C1 (Clontech) as a template, with a sense primer containing an EcoRI recognition site (5'CGGAATTCCGTGAGCAAGGGCGAGGAGC3') and an antisense primer containing an $\mathrm{XbaI}$ recognition site (5'GCTCTAGATCCGGTGGATCC3'). The fragment was double digested with EcoRI-XbaI and cloned into the EcoRI-XbaI sites of pCS2+NLS+MT (pCS2+NLS+MT-GFP). This vector contains the SV40 large T antigen NLS followed by six copies of the myc (MT) epitope (Rupp et al., 1994). Two complementary 
oligonucleotides (sense, 5'CTAGACAGGGTTGTATGGGATTGCCATGTGTGGTGATGTAA3'; antisense, 5'TTACATCACCACACATGGCAATCCCATACAACCCTGT3') encoding the last 11 codons of human N-Ras were annealed, phosphorylated, purified with the use of a nucleotide removal kit (Qiagen, Hilden, Germany), and cloned into the XbaI-SnaBI sites of pCS2+NLS+MT-GFP (pCS2+NLS+MT-GFP-N-Ras).

\section{RNA Techniques}

For RNA synthesis, plasmid templates were linearized with NotI. Capped RNAs were synthesized with a MessageMachine kit (Ambion, Austin, TX). RNAs were purified with the use of spin columns (Qiagen) and quantitated by reading the absorbance at $260 \mathrm{~nm}$.

RNA from oocytes and embryos (10 per extraction) was extracted as described (Stick, 1988). RNAs were treated with $200 \mathrm{U} / \mathrm{ml}$ DNase I for $30 \mathrm{~min}$ at $37^{\circ} \mathrm{C}$ and purified with the use of spin columns.

First-strand synthesis $(20 \mu \mathrm{l})$ for RT-PCR was done with $2 \mu \mathrm{g}$ of total RNA with a SuperScript preamplification system (GIBCO-BRL, Gaithersburg, MD) and oligo(dT) primers according to the manufacturer's instructions, including RNase $\mathrm{H}$ treatment and heat inactivation at the end of the reaction. PCR amplification $(20 \mu \mathrm{l})$ was carried out with one-third of the first-strand reaction with $10 \mathrm{pmol}$ of each oligonucleotide primer, $0.5 \mathrm{U}$ of AmpliTaq-Gold polymerase, and the corresponding buffer (Perkin Elmer-Cetus, Norwalk, $\mathrm{CT})$ in the presence of $1.25 \mathrm{mM} \mathrm{MgCl}$. Cycling parameters were initial denaturation $\left(10 \mathrm{~min}, 95^{\circ} \mathrm{C}\right)$, followed by 30 cycles $\left(30 \mathrm{~s}, 95^{\circ} \mathrm{C}\right.$; $\left.30 \mathrm{~s}, 55^{\circ} \mathrm{C} ; 30 \mathrm{~s}, 68^{\circ} \mathrm{C}\right)$, and a final polymerization step $(10 \mathrm{~min}$ $68^{\circ} \mathrm{C}$ ). Reaction products were separated on $1.5 \%$ agarose gels and stained with ethidium bromide. The following primers were used: sense primer lamin B3, 5'GGGCTGCTGGTGCTGGTGCTG3'; antisense primer B3a, 5'AATTACATGATGGAACAGC3'; antisense primer B3b, 5'AGCCATTATGAAACTGAAC3'; sense primer lamin B1 (GenBank accession number X06344), 5'CCCGTATTCACATGATGGCGC3'; antisense primer B1, 5'GGCCACACTGAAGAATTCTCAGGG3'; sense primer lamin B2 (GenBank accession number X54099), 5'CATGACAGAGCAGCCTCTGG3'; and antisense primer B2, 5'GAGTTCCTGGGGTACAGGCAGC3'.

\section{Preparation of Egg Homogenates}

Xenopus eggs were washed several times in homogenization buffer (10 mM HEPES-KOH, $100 \mathrm{mM} \mathrm{KCl}, 1 \mathrm{mM} \mathrm{MgCl} 2,50 \mathrm{mM}$ sucrose, pH 7.7) (Matthews and Colman, 1991). Eggs were packed by centrifugation at $100 \mathrm{rpm}$ at $4^{\circ} \mathrm{C}$ for $60 \mathrm{~s}$ in a bench-top centrifuge, buffer was removed, and the eggs were crushed by centrifugation at $14,000 \times g$ for $8 \mathrm{~min}$ at $4^{\circ} \mathrm{C}$. The layer between the yolk pellet and the lipid cap was gathered and used immediately for fractionation or floating gradient centrifugation or was shock frozen in liquid nitrogen and stored at $-70^{\circ} \mathrm{C}$.

\section{Fractionation of Egg Extract}

Twenty microliters of egg homogenate was diluted with $100 \mu \mathrm{l}$ of egg buffer (10 mM HEPES-KOH, $50 \mathrm{mM} \mathrm{KCl}, 20 \mathrm{mM} \beta$-glycerophosphate, $2 \mathrm{mM} \mathrm{MgCl}_{2}, 1 \mathrm{mM}$ DTT, $1 \mathrm{mM}$ EGTA, $1 \mathrm{mM}$ ATP, pH 7.5). Samples were centrifuged in a TL100.3 rotor (Beckmann, Fullerton, CA) at $200,000 \times g$ for $45 \mathrm{~min}$ at $4^{\circ} \mathrm{C}$. The resulting supernatants (S1) were removed. The pellets were carefully washed with $100 \mu \mathrm{l}$ of egg buffer and centrifuged for $15 \mathrm{~min}$ (see above), and the resulting supernatants were discarded. The pellets were resuspended in $100 \mu \mathrm{l}$ of egg buffer containing $4 \mathrm{M}$ urea, incubated on ice for $10 \mathrm{~min}$, and centrifuged for $45 \mathrm{~min}$ as described above. Supernatants (S2) were removed, and the pellets were resuspended directly in $100 \mu \mathrm{l}$ of egg buffer containing 1\% NP40, incubated, and centrifuged as described above, giving rise to supernatants (S3) and pellets (P3). The supernatants were concentrated by precipitation with methanol $/ \mathrm{CHCl}_{3} / \mathrm{H}_{2} \mathrm{O}$ (Wessel and Flügge, 1984). The pellets and the precipitates of the supernatants were dissolved in SDSPAGE sample buffer and boiled for $4 \mathrm{~min}$.

\section{Floating Gradient Analysis}

Egg homogenates were mixed with 1 volume of egg buffer. Up to 50 $\mu \mathrm{l}$ of the diluted homogenate was made $1.3 \%$ sucrose with a $2.3 \mathrm{M}$ sucrose stock in egg buffer. This homogenate was overlaid with 300 $\mu \mathrm{l}$ each of $1.1 \mathrm{M}, 0.9 \mathrm{M}$, and $0.7 \mathrm{M}$ sucrose in egg buffer in a 2.2-ml polyallomer tube (Beckman). Gradients were centrifuged in a TLS-55 rotor (Beckman) at 200,000 $\times g$ for $2 \mathrm{~h}$ at $4^{\circ} \mathrm{C}$. Fractions of 75 $\mu \mathrm{l}$ were taken from the top. Twenty microliters of each fraction was mixed with $2 \times$ SDS-PAGE sample buffer and boiled for $4 \mathrm{~min}$.

\section{In Vitro Translation and Immunoprecipitation}

Lamins were translated in vitro in a rabbit reticulocyte coupled transcription/translation system with the use of SP6 RNA polymerase (Promega, Madison, WI). To achieve sufficient geranylgeranyl incorporation, the reticulocyte lysate was supplemented (after $2 \mathrm{~h}$ of incubation at $30^{\circ} \mathrm{C}$ ) with 1 volume of freshly prepared undiluted egg homogenate supplemented with $8 \mathrm{mM}$ phosphocreatine, $1 \mathrm{mM}$ spermidine, $50 \mu \mathrm{g} / \mathrm{ml}$ cytochalasin B, and $1 \mu \mathrm{g} / \mathrm{ml}$ aprotinin (Matthews and Colman, 1991). For radiolabeling, $10 \mu \mathrm{Ci}$ of $\left[{ }^{3} \mathrm{H}\right]$ mevalonolactone (20-40 Ci/mmol; New England Nuclear, Köln, Germany) or $\left[{ }^{3} \mathrm{H}\right]$ geranylgeranylpyrophosphate $(10-30 \mathrm{Ci} / \mathrm{mmol}$; New England Nuclear) was used per reaction. The radioactive reagents were dried by vacuum centrifugation and dissolved in egg extract before addition to the reticulocyte lysate. The mixture was incubated for $1 \mathrm{~h}$ at $25^{\circ} \mathrm{C}$.

Immunoprecipitation was as described (Stick, 1988) with antiFlag mAb M2 (Kodak, Rochester, NY) with the use of GammaBind Sepharose (Pharmacia, Freiburg, Germany) and wash buffer $(20 \mathrm{mM}$ HEPES-KOH, $150 \mathrm{mM} \mathrm{NaCl}, 5 \mathrm{mM}$ EDTA, 1\% NP-40, $50 \mathrm{mM}$ sucrose, pH 7.2, and protease inhibitor tablets; Roche, Mannheim, Germany).

\section{Electrophoresis and Immunoblotting}

Isoelectric focusing (IEF), SDS-PAGE, and immunoblotting were done as described (Huttenlauch et al., 1998). Immobilized $\mathrm{pH}$ gradient buffer 3-10 NL (0.4\%; Pharmacia) was used for IEF gels. In some experiments, Immobilon polyvinylidene difluoride membranes (Millipore, Bedford, MA) were used instead of nitrocellulose.

\section{Tissue Culture and Cell Lines}

HeLa and MCF7 (HTB-22; American Type Culture Collection, Rockville, $\mathrm{MD}$ ) cells were maintained in $5 \% \mathrm{CO}_{2}$ at $37^{\circ} \mathrm{C}$ in Dulbecco's modified MEM containing 10\% FCS. For transfections, cells were grown on coverslips in $3.5-\mathrm{cm}$ dishes. Cells were transfected with $0.5-1 \mu \mathrm{g}$ of DNA per dish with the use of FuGene 6 (Roche) according to the manufacturer's instructions.

Cells were fixed in 4\% formaldehyde in PBS for $20 \mathrm{~min}$, followed by $4 \%$ formaldehyde, $0.5 \%$ Triton in PBS for $10 \mathrm{~min}$, counterstained with $0.1 \mu \mathrm{g} / \mathrm{ml} \quad 4^{\prime}, 6^{\prime}$-diamidino-2-phenyl-indol-dihydrochloride (Roche) for $2 \mathrm{~min}$, washed with PBS, mounted in Elvanol (Hoechst, Frankfurt, Germany), and viewed with a LSM 510 confocal laser scanning microscope (Zeiss, Obarkochen, Germany).

\section{Software Applications}

X-ray films were scanned with a Duoscan T2500 (Agfa, Köln, Germany). Digital images (laser scan images and scans of x-ray films) were processed with LSM software (Zeiss), Photoshop 4.0 (Adobe, Mountain View, CA), and FreeHand 8.0 (Macromedia, San Francisco, CA). 


\section{RESULTS}

\section{Expression of Two Variants of Lamin B3 in Oocytes} and Embryos of Xenopus

Lamin B3 is the major lamin of amphibian oocytes (Stick and Krohne, 1982). It serves as a maternal lamin pool for the formation of the cleavage nuclei up to the mid blastula. After mid blastula, B3 is gradually replaced by the newly synthesized lamins B1 and B2, and in later development it represents only a minor lamin (Benavente et al., 1985; Stick and Hausen, 1985). The Xenopus lamin B3 gene encodes two CaaX-carrying exons that give rise to two mRNAs via alternative splicing (Figure 1). These mRNAs code for two B3 proteins, named lamin B3a and B3b, that differ only in their $12 \mathrm{COOH}$-terminal residues (Table 1) (Döring and Stick, 1990). Both B3 mRNAs are present in oocytes, but mRNA $\mathrm{B} 3 \mathrm{a}$ is the predominant species, as revealed by RNase protection assay (Döring and Stick, 1990).

We used RT-PCR to monitor the presence of the two mRNAs in early development. A scheme of the relevant part of the lamin B3 gene and the two alternatively spliced RNAs, with the positions of the three primers used in the RT-PCR, is shown in Figure 1A. PCR with a $5^{\prime}$ primer common to both B3 mRNAs, in combination with a B3b-specific $3^{\prime}$ primer, gives rise to a single amplicon of the expected size of 275 base pairs. The level of B3b mRNA is constant in oocytes and early embryos but shows a significant increase in tadpole stages (Figure 1B, B3b, lane 5). The level of lamin B3a mRNA is more variable in early development. In tadpole stages, B3a mRNA is hardly detectable (Figure 1B, B3a, lane 5). The slower-migrating band in Figure 1B (B3a, lane 5) represents an amplification product of lamin B3b mRNA with the B3a-specific primer (Figure 1A). Under our amplification conditions, this band is detected only when mRNA $\mathrm{B} 3 \mathrm{~b}$ is present at sufficiently high concentrations. The levels of mRNAs encoding lamin B1 and B2 were also analyzed and appeared constant throughout development (Figure 1B, B1 and B2). The identities of the individual amplicons shown in Figure 1 were verified by subcloning and sequencing. In summary, mRNA B3a is the predominant lamin B3 mRNA in oocytes (Döring and Stick, 1990). Both B3 mRNA variants can be detected in early development. In tadpoles, mRNA B3b is up-regulated, whereas mRNA B3a is no longer detectable.

None of the lamin B3-specific antibodies available at present allows discrimination between the two B3 variants. However, two-dimensional gel electrophoresis can separate the two variants, because lamin B3b is more basic than lamin $\mathrm{B} 3 \mathrm{a}$ as a result of the presence of a basic cluster within the B3b-specific exon (Table 1). Therefore, we separated total oocyte nuclear proteins by two-dimensional gel electrophoresis and detected lamin B3 polypeptides with a B3specific $\mathrm{mAb}$. A nonlinear $\mathrm{pH}$ gradient was chosen in the first dimension to optimize resolution between $\mathrm{pH} 5.0$ and 6.0. Several major lamin B3 spots with isoelectric points between $\mathrm{pH} 5.55$ and 5.65 as well as a minor spot with a significantly more basic isoelectric point of 5.75 were detected [Figure 2, B3 (GV)]. Although the amount of protein of the minor spot was clearly detectable by immunological methods, it was too low to allow direct identification of this spot as either lamin B3a or B3b by protein analytical methods. To allow an assignment of the spots, we generated
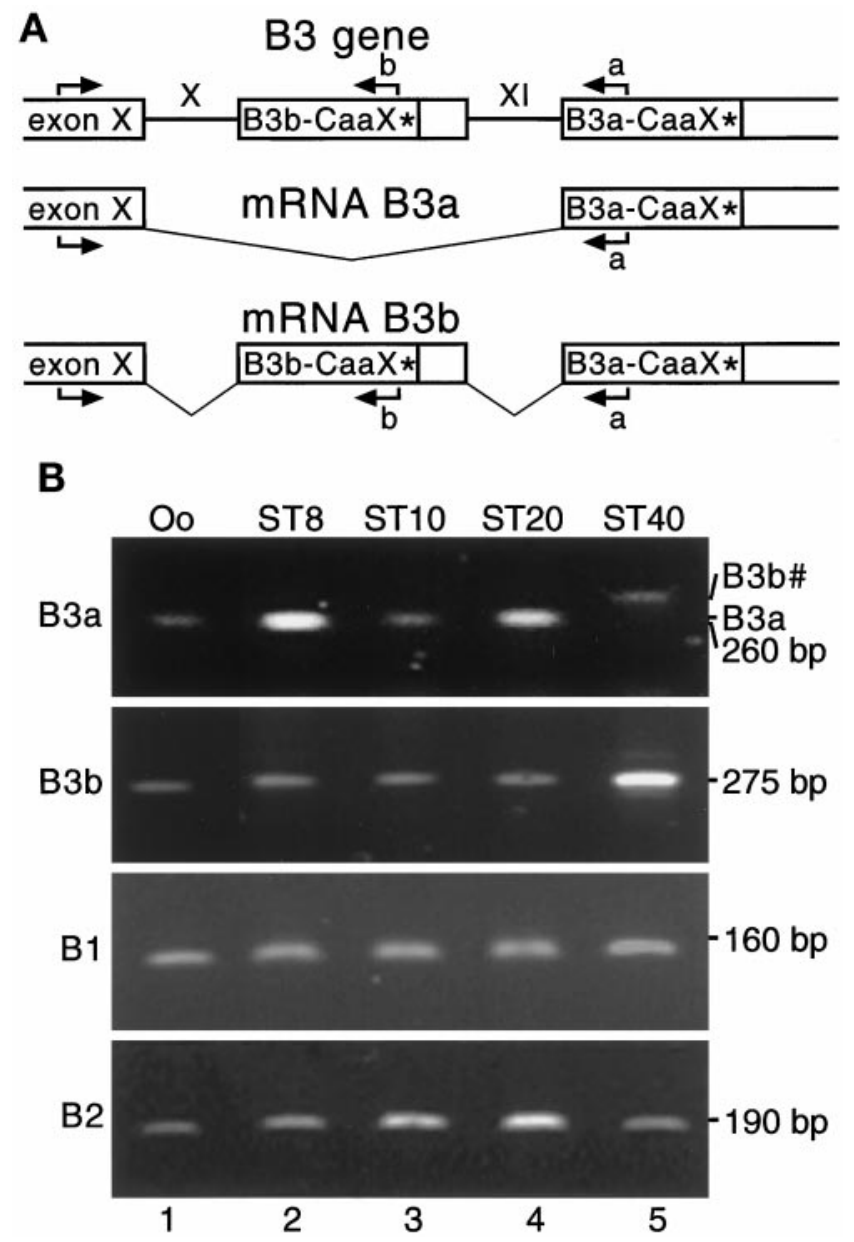

Figure 1. Differential expression of two alternatively spliced lamin B3 mRNAs in Xenopus oocytes and embryos. (A) A scheme of the relevant region of the gene encoding Xenopus laevis lamin B3 and schemes of the two mRNAs that are generated by alternative splicing are shown. Exons are drawn as boxes, introns as lines, and spliced regions as thin V-shaped lines. Stars depict termination codons, and arrows mark the positions and the directions of the oligonucleotide primers used in RT-PCR. (B) Total RNA of oocytes (Oo) and different embryonic stages (ST8-ST40) was used for RTPCR with primers specific for mRNA encoding lamin B3a (B3a), lamin B3b (B3b), lamin B1 (B1), or lamin B2 (B2). Ethidium bromidestained agarose gels are shown. Size markers are indicated at the right. In panel $\mathrm{B} 3 \mathrm{a}$, the positions of amplicons resulting from amplification of either mRNA B3a (B3a) or mRNA B3b (B3b\#) with B3a specific primer is indicated (see scheme in A for explanation).

lamin B3a and B3b constructs that carried a Flag epitope at their $\mathrm{NH}_{2}$ termini. Synthetic RNA encoding either of these epitope-tagged lamins was injected into the cytoplasm of oocytes. Oocytes were incubated for $12 \mathrm{~h}$ to allow synthesis and targeting of lamin polypeptides. Both epitope-tagged lamin variants were efficiently accumulated in the nucleus and incorporated into the NE lamina, as judged by gel electrophoretic separation and immunoblotting of isolated cytoplasm, nuclei, and nuclei fractionated into nuclear content and NE. Their spatial distribution within the NE was 
Table 1. COOH-terminal sequences of proteins used in this study

\begin{tabular}{|c|c|}
\hline Name & Sequence \\
\hline Lamin B3a & S H Q S V D P S C S I M \\
\hline Lamin B3b & TKRRKKKC $\overline{C S V S}$ \\
\hline $\mathrm{B} 3 \mathrm{aCC}$ & S H Q S V D P C $\overline{C S ~ I ~ M ~}$ \\
\hline B3b3Q & T Q Q Q K K KC C V S \\
\hline $\mathrm{B} 3 \mathrm{~b} 6 \mathrm{Q}$ & $\mathrm{T} \mathbf{Q} \mathbf{Q} \mathbf{Q} \mathbf{Q} \mathbf{Q} \mathbf{Q} \overline{C S V S}$ \\
\hline B3bSC & T K R R K K K S C S V S \\
\hline $\mathrm{B} 3 \mathrm{bCS}$ & TKRRKKKC $\overline{\text { S V V }}$ \\
\hline B3aCAIL & S HQS V D P S $\overline{C \mathbf{A I L}}$ \\
\hline GFP-N-Ras & - Q G C M G L P $\overline{\mathrm{CVV} M}$ \\
\hline
\end{tabular}

indistinguishable from that of the endogenous lamin B3, as revealed by immunofluorescence microscopy of isolated NEs.

Total nuclear proteins of injected oocytes were separated by two-dimensional gel electrophoresis, and the epitopetagged lamins were detected with mAb M2, which is specific

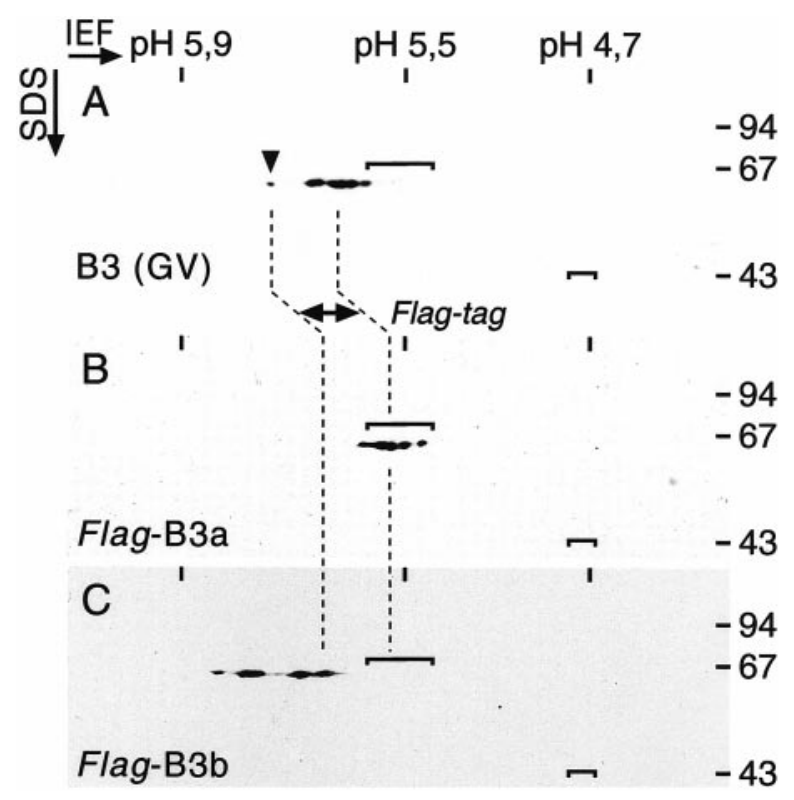

Figure 2. Two variants of lamin B3 are expressed in Xenopus oocytes. Three nuclei each of noninjected oocytes (A) or oocytes injected with RNA encoding lamin Flag-B3a (B) or Flag-B3b (C) were manually isolated, and proteins were separated by IEF in the first dimension (IEF) and by SDS-PAGE in the second dimension (SDS). Lamins were detected after immunoblotting with the use of either mAb L6-5D5, which recognizes lamin B3 [B3 (GV)], or mAb M2, which recognizes the Flag epitope (Flag-B3a and Flag-B3b). The shift in isoelectric point of the two epitope-tagged lamins, which is due to the presence of the Flag epitope, is indicated by broken lines. Note the higher resolution of the IEF gel in the range between $\mathrm{pH} 5.7$ and 5.8 caused by the nonlinearity of the $\mathrm{pH}$ gradient. Isoelectric points (averages) of standard proteins in the first dimension (left to right: phosphorylase $b, \mathrm{BSA}$, and ovalbumin) are given at the top. The positions of the isoelectric variants of BSA and ovalbumin are indicated by brackets within each blot. The sizes of molecular mass markers in the second dimension are given in kilodaltons at the right. for the Flag epitope. The resulting pattern was compared with that of endogenous lamin B3 (Figure 2A). Because lamins are phosphorylated during interphase, they give rise to multiple spots in the IEF dimension depending on the degree of phosphorylation (Gerace and Blobel, 1980; Ottaviano and Gerace, 1985). The pattern of isoelectric variants of Flag-B3a polypeptides (Figure 2B) is remarkably similar to that of the major spots of the endogenous lamin B3 (Stick and Hausen, 1985; Stick, 1987). In contrast to the endogenous lamin, a spot at a more basic $\mathrm{pH}$ was never observed, even after overexposure of the blots. However, the Flag-B3a spots are shifted by $\sim 0.1 \mathrm{pH}$ unit. This shift is due to the presence of the Flag epitope that introduces net negative charge (for sequence, see MATERIALS AND METHODS). Our observation indicates that the major spots of the endogenous lamin B3 represent isoelectric variants of lamin B3a.

Flag-B3b shows several major spots in the range between pH 5.6 and 5.85 (Figure 2C). The most acidic of these spots corresponds to the minor basic spot of the endogenous lamin B3, if the $\mathrm{pH}$ shift caused by the Flag epitope is taken into account (Figure 2, A and C). This indicates that the minor basic spot of endogenous lamin B3 represents lamin $\mathrm{B} 3 \mathrm{~b}$. The presence of additional, more basic spots of FlagB3b (Figure 2C) might be due to inefficient phosphorylation of the relatively high amounts of Flag-B3b introduced by RNA injection. Moreover, the rather broad separation of these isoelectric variants is due to the nonlinearity of the $\mathrm{pH}$ gradient, which is very shallow between $\mathrm{pH} 5.7$ and 5.8 .

In conclusion, both splice variants of lamin B3 are expressed at the protein level in oocytes. Lamin B3a is the quantitative major B3 lamin, whereas B3b is a minor component. This correlates well with the relative abundance of the mRNAs encoding the two variants (Döring and Stick, 1990).

\section{Lamin B3a and B3b Differ in Their Affinity to Membranes}

Lamin B3a and B3b differ in their last exon (Döring and Stick, 1990), which contains the CaaX tetrapeptide (Table 1). The CaaX motif is necessary for membrane association. It has been shown that, in addition to the CaaX-dependent modifications, either a basic cluster or palmitoylation of additional cysteine residue(s) is required to localize Ras to the PM (Hancock et al., 1990; Dudler and Gelb, 1996). The lamin B3b-specific exon contains both a basic cluster and an additional cysteine residue adjacent to the CaaX motif (Table 1). Therefore, it was analyzed to determine whether the two lamin splice variants differ in their membrane association. Flag-tagged versions of either splice variant were expressed in oocytes by injection of synthetic RNA. Injected oocytes were matured to eggs by incubation with progesterone, and extracts, containing membranes as well as soluble proteins, were prepared. The extracts were fractionated by highspeed centrifugation into a supernatant containing soluble proteins and a pellet containing membrane vesicles and large protein complexes. The pellet was extracted with $4 \mathrm{M}$ urea and, after another round of centrifugation, with $1 \%$ NP-40. In this way, three supernatant fractions and a final pellet fraction were obtained (see MATERIALS AND METHODS). The fractions were probed for epitope-tagged lamin variants by immunoblotting after SDS-PAGE. The majority of lamin Flag-B3a was found in the first superna- 


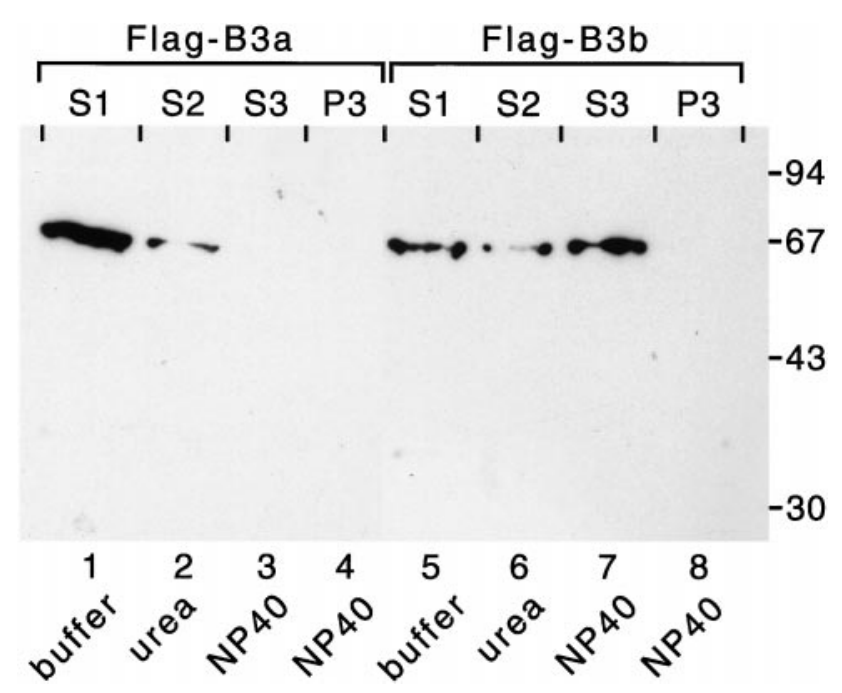

Figure 3. Differential extraction of egg membranes of Xenopus expressing either lamin Flag-B3a or Flag-B3b. Oocytes were injected with RNA encoding either lamin Flag-B3a or Flag-B3b. Oocytes were matured to eggs with progesterone, and egg homogenates were prepared by low-speed centrifugation. Extracts were diluted in buffer (see MATERIALS AND METHODS) and separated into a supernatant (S1) and a pellet. The pellet was resuspended in buffer containing $4 \mathrm{M}$ urea and separated again into a supernatant (S2) and a pellet. This pellet was resuspended in buffer containing $1 \% \mathrm{NP}-40$ and separated into a supernatant (S3) and a pellet (P3). The fractions were separated by SDS-PAGE, and epitope-tagged lamins were detected by immunoblotting with mAb M2. The sizes of molecular mass markers are given in kilodaltons at the right.

tant fraction of soluble proteins (Figure 3, lane 1). Only a minor amount of Flag-B3a was found in the pellet. This was completely soluble in $4 \mathrm{M}$ urea, as shown after the second centrifugation (Figure 3, lane 2). Consequently, Flag-B3a was not found in fractions obtained after further treatment with NP-40 (Figure 3, lanes 3 and 4). This indicates that lamin Flag-B3a is soluble in eggs and that only a small fraction is either loosely attached to membranes or exists in large urea-soluble protein complexes. The fractionation behavior of lamin Flag-B3b was significantly different. Only a relatively small fraction of Flag-B3b was soluble (Figure 3, lane 5). A comparable fraction to that of Flag-B3a was extracted with $4 \mathrm{M}$ urea (Figure 3, compare lanes 2 and 6), but the majority of Flag-B3b could be sedimented after urea treatment. Flag-B3b in this fraction was completely solubilized after treatment with NP-40 (Figure 3, lanes 7 and 8), indicating that in the egg, the majority of lamin Flag-B3b might be membrane bound.

Sedimentation analysis could not unequivocally discriminate between sedimentation attributable to membrane association and sedimentation caused by association with large protein complexes. Therefore, floating sucrose gradient centrifugation was used to determine whether lamin Flag-B3b is associated with membranes. In this procedure, cell extracts are brought to a high concentration of sucrose and are then overlaid with buffers of decreasing sucrose concentrations. Upon centrifugation, lipid vesicles and proteins bound to these vesicles float according to their buoyant density, whereas soluble proteins remain at the bottom (Wil- son and Newport, 1988; Meier and Georgatos, 1994). In this way, a small amount of membrane-bound protein can readily be separated from the soluble fraction even if the latter is in large excess. First, total extracts of eggs were analyzed that had been matured from uninjected oocytes. Aliquots of the gradient fractions were separated by SDSPAGE, and lamin B3 was detected by immunoblotting with a lamin B3-specific mAb (Figure 4A). The vast majority of lamin B3 remained at the bottom of the gradient within the loading zone. Only a small portion of B3 floated to lower sucrose concentrations, indicating that only a minor fraction of the endogenous lamin B3 was stably associated with membranes in eggs. This is in agreement with previous findings (Firmbach-Kraft and Stick, 1993; Lourim and Krohne, 1993).

\section{A Basic Cluster and an Extra Cysteine Mediate Membrane Association of Lamin B3b}

Next, lamin Flag-B3a and Flag-B3b as well as several mutants of the two variants were analyzed. Mutants were generated by site-directed mutagenesis. Selection of the mutated residues was guided by results obtained with Ras proteins (Hancock et al., 1990). We focused our attention on the extra cysteine residue as a potential palmitoylation site and on the basic cluster. The sequences of the last exon of wild-type and mutant lamin B3 variants are listed in Table 1.

Lamin Flag-B3a remained exclusively soluble as determined by floating gradient analysis (Figure 4B). In contrast, Flag-B3b was predominantly membrane associated. Only a minor fraction of Flag-B3b remained within the loading zone (Figure 4C). Flag-B3b was also analyzed by gradient centrifugation in the presence of nonionic detergent. Under these conditions, membranes are dissolved. Flag-B3b remained completely within the loading zone, demonstrating that flotation was dependent on the presence of lipid membranes.

Next, we studied the contribution to membrane association of the extra cysteine residue on the one hand and the basic cluster on the other. Introduction of a single extra cysteine residue into lamin Flag-B3a (at the position where a cysteine is found in lamin B3b) (Flag-B3aCC) resulted in membrane binding. Flag-B3aCC was nearly completely ( $80-90 \%)$ membrane associated (Figure 4D). Replacement of the extra cysteine with a serine residue in Flag-B3b (Flag-B3bSC) drastically reduced membrane association (Figure $4 \mathrm{G}$ ). The influence of the basic cluster was much less dramatic. Replacement of the first three basic residues by glutamine residues (Flag-B3b3Q) had no significant effect on membrane association (Figure 4E), whereas replacement of the entire basic cluster by glutamine residues (Flag-B3b6Q) led to a moderate decrease of membrane association (Figure 4F).

The effect of palmitoylation or a basic cluster on membrane association strictly depends on the CaaX-dependent modifications (Hancock et al., 1989, 1990; Dudler and Gelb, 1996). As a control, the CaaX cysteine residue of Flag-B3b was replaced with a serine residue (Flag-B3bCS) so that this mutant protein could not be isoprenylated. As expected, Flag-B3bCS was completely soluble in eggs (Figure $4 \mathrm{H}$ ). This experiment demonstrates the efficiency with which soluble lamins can be separated from membrane-associated lamins by floating gradient centrifugation. Consequently, the presence of even small amounts of lamin B3 in the flotation zone indicates stable membrane association of this lamin fraction 


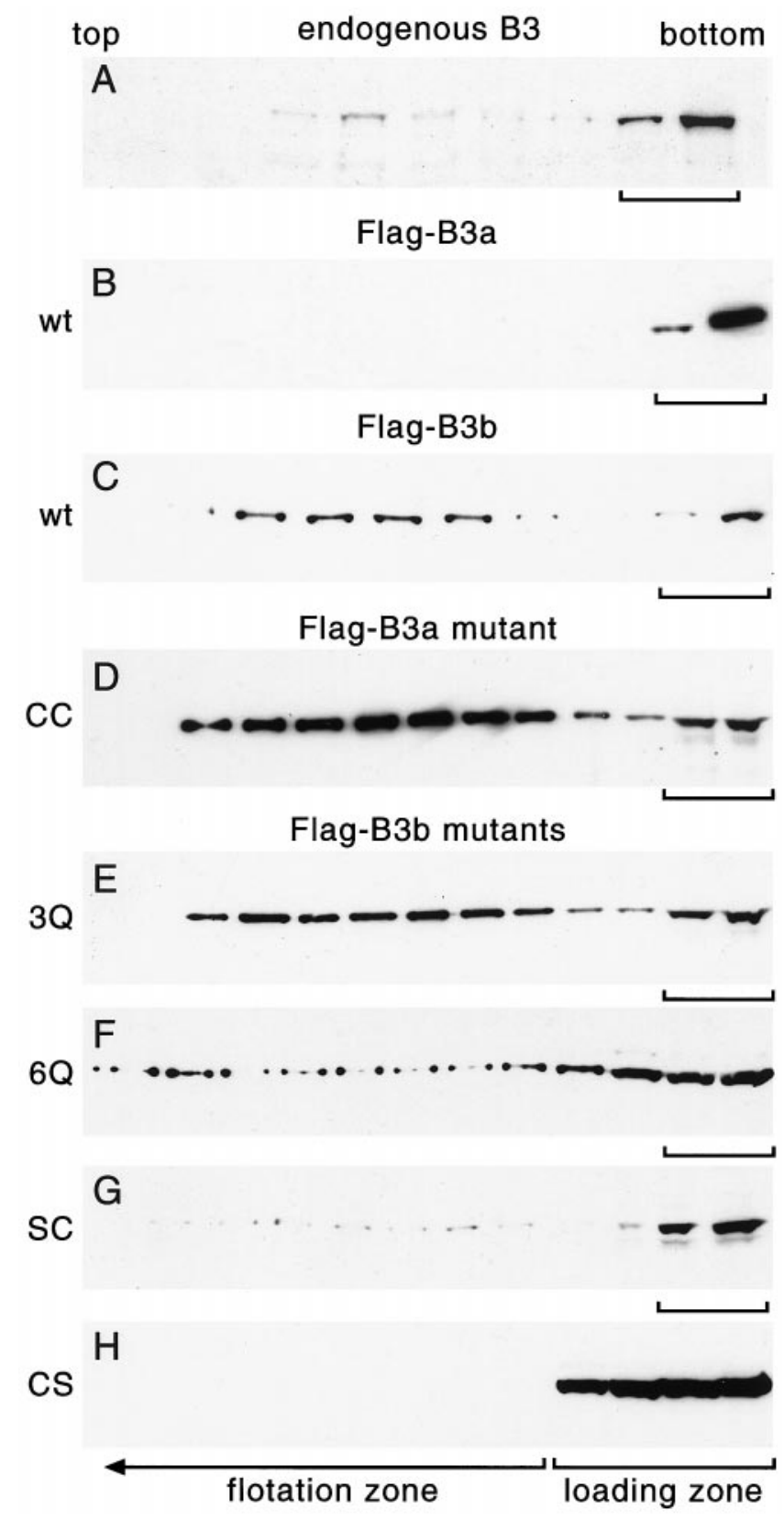

Figure 4. Analysis of membrane association of endogenous and mutant lamin B3 variants by floating gradient centrifugation. Wildtype and mutant lamin B3 variants were expressed in oocytes by RNA injection. Oocytes were matured to eggs with progesterone, and egg homogenates were prepared by low-speed centrifugation. Membranes were separated from soluble proteins by floating gradient centrifugation of egg homogenates (see MATERIALS AND METHODS). Aliquots of the gradient fractions were separated by SDS-PAGE, and lamins were detected by immunoblotting with $\mathrm{mAb}$ L6-5D5 (A) or mAb M2 (B-H). The loading zones in A-H and the flotation zone in $\mathrm{H}$ are indicated by brackets. Note that the two fractions of the bottom of gradients B-H are not shown. The names of the lamin B3 constructs are given at left. The sequences of the last exon of these constructs are listed in Table 1.
(Figure 4A). In contrast to the sedimentation analysis (Figure $3)$, floating gradient analysis could uncover true membrane binding.

These results suggest that $\mathrm{B} 3 \mathrm{a}$ and $\mathrm{B} 3 \mathrm{~b}$ constitute the soluble and membrane-associated fractions, respectively, of lamin B3 in eggs. Furthermore, they show that an extra cysteine residue adjacent to the CaaX motif is crucial for association with inner NE-derived membranes, suggesting that palmitoylation plays an important role in lamin B3 membrane association in oocytes and eggs.

The two Flag lamin variants were also expressed in enucleated oocytes by RNA injection, and their membrane partition was analyzed by flotation gradient centrifugation. Neither Flag-B3a nor Flag-B3b associated with endomembranes. This could be explained in one of two ways. Either palmitoylation of lamin B3b occurs after translocation into the nucleus, or the palmitoylated lamin is prevented from binding to cytoplasmic membranes by interaction with unknown escort protein(s).

\section{Geranylgeranylation Does Not Alter the Membrane Association of Lamin B3a in Eggs}

CaaX-containing proteins can be either farnesylated or geranylgeranylated. The type of isoprene residue is determined by the COOH-terminal residue of the CaaX motif (Reiss et al., 1991; Schafer and Rine, 1992). Ras proteins and all nuclear lamins are farnesylated. Mutational analysis has shown that geranylgeranylated Ras proteins show a significantly higher affinity with membranes than the farnesylated wildtype proteins (Hancock et al., 1991b). Therefore, the CaaX motif CSIM of lamin Flag-B3a was changed to CAIL, the CaaX motif of a brain $G$ protein $\gamma$-subunit that is geranylgeranylated (Flag-B3aCAIL) (Hancock et al., 1991b). The specificity of the Flag-B3aCAIL modification was demonstrated in an in vitro translation system. The rabbit reticulocyte translation system was supplemented with Xenopus egg extract to reach sufficient incorporation of radiolabeled geranylgeranyl moieties. Both polypeptides were efficiently translated in vitro as detected by immunoblotting with an anti-Flag mAb (Figure 5A, lanes 1 and 2), and both were isoprenylated in the presence of $\left[{ }^{3} \mathrm{H}\right]$ mevalonic acid, a precursor of isoprene synthesis (Figure 5A, lanes 5 and 6). Two closely spaced bands were detected in the immunoblot of the Flag-B3aCAIL mutant (Figure 5A, lane 2). Comparison of the immunoblot and the autoradiogram showed that the faster-migrating band of Flag-B3aCAIL was isoprenylated (Figure 5A, compare lanes 2 and 6), whereas the slowermigrating band represented the unmodified form. Only Flag-B3aCAIL was efficiently geranylgeranylated (Figure 5A, lane 4 ), whereas the wild-type protein was a very poor substrate for the geranylgeranyltransferase (Figure 5A, lane 3).

After having established that lamin Flag-B3aCAIL is specifically geranylgeranylated, the membrane affinity of this construct was tested in the oocyte/egg system with the floating gradient method. As in the vitro translation system, modified and unmodified Flag-B3aCAIL was detected in egg extracts by immunoblotting. Significantly, both the geranylgeranylated and the unprocessed forms remained completely within the loading zone of the gradient, indicating that geranylgeranylation is not sufficient for stable binding of lamin B3a to NE-derived membranes (Figure 5B). 


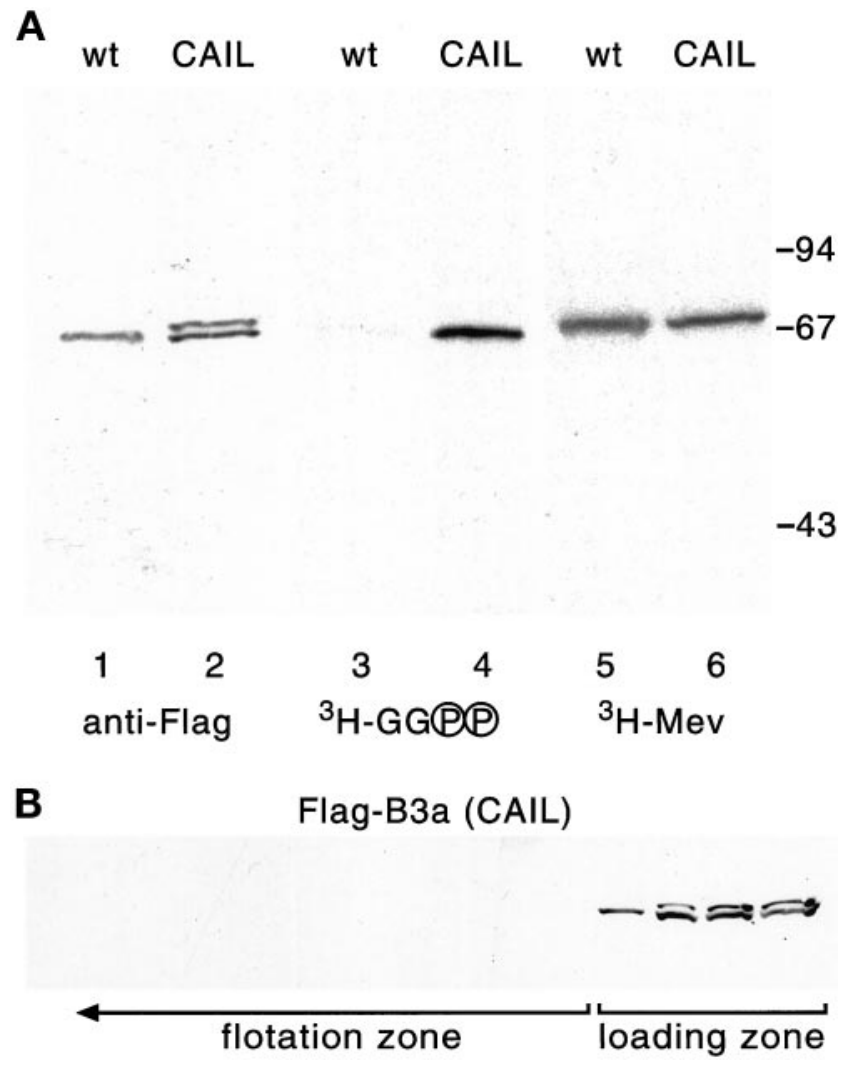

Figure 5. Geranylgeranylation is not sufficient for stable binding of lamin B3a to NE-derived membranes. (A) Lamin Flag-B3a (wt) and Flag-B3aCAIL (CAIL) were translated in a rabbit reticulocyte lysate supplemented with Xenopus egg extract in the presence of $\left[{ }^{3} \mathrm{H}\right]$ geranylgeranylpyrophosphate $\left({ }^{3} \mathrm{H}-\mathrm{GG}-\mathrm{PP}\right)$ or $\left[{ }^{3} \mathrm{H}\right]$ mevalonolactone $\left({ }^{3} \mathrm{H}-\right.$ Mev). Lamins were detected after SDS-PAGE and immunoblotting with $\mathrm{mAb}$ M2 (anti-Flag) or after immunoprecipitation with $\mathrm{mAb}$ M2 and SDS-PAGE. For autoradiography, gels were blotted to nitrocellulose and exposed to $\mathrm{x}$-ray film for $5 \mathrm{~d}\left({ }^{3} \mathrm{H}\right.$-GG-PP) and $10 \mathrm{~d}$ $\left({ }^{3} \mathrm{H}-\mathrm{Mev}\right)$. The upper band in lane 2 represents unmodified Flag$\mathrm{B} 3 \mathrm{aCAIL}$ protein. The sizes of molecular mass markers are given in kilodaltons at the right. (B) Lamin Flag-B3aCAIL was expressed in oocytes by RNA injection. Membrane association in eggs was analyzed as described in the legend to Figure 4. Note that in the oocyte/egg system, a fraction of Flag-B3aCAIL remains unmodified.

\section{Association of Proteins with the PM and the Inner NE Is Mediated by the Same Targeting Motifs}

The sequence motifs that target Ras proteins to and mediate stable association with the PM are remarkably similar to those that mediate stable association of lamin B3b with the inner nuclear membrane in Xenopus eggs. Targeting of lamins to the nucleus is mediated by a NLS that is present in the lamin tail domain. The membrane-targeting signals, therefore, might direct a protein to either the PM or the inner nuclear membrane depending on whether they act in the absence or presence of a NLS. This hypothesis was tested by transiently expressing chimeric GFP-lamin constructs in human tissue culture cells. These constructs contained the tail domain of wild-type or mutant lamin B3 variants fused to the $\mathrm{COOH}$ terminus of GFP. Two types of constructs were analyzed. One type contained the functional NLS of the lamin tail domain (Figure 6, A-F). In the other type, the NLS was inactivated by site-directed mutagenesis (Figure 6, G-L) (see MATERIALS AND METHODS). The constructs were expressed in either HeLa or MCF7 cells by transfection. The subcellular distribution of GFP fluorescence was analyzed by confocal laser scan microscopy of formaldehyde-fixed cells. Identical results were obtained with both cell lines. Cells were observed 24 and $48 \mathrm{~h}$ after transfection. No differences in the subcellular distribution were noted at the two time points. Cells transfected with GFP alone showed diffuse staining throughout the entire cytoplasm and nucleoplasm, with no preferential staining of membranes.

GFP-B3 chimeras that contained an intact NLS gave rise to three different staining patterns. Three constructs, GFPB3awt, GFP-B3aCC, and GFP-B3bCS, were exclusively nucleoplasmic. They showed no association with the NE (Figure 6, A, B, and F). GFP-B3b6Q and GFP-B3bSC were also nuclear, but they showed both nucleoplasmic and nuclear rim staining (Figure 6, D and E). GFP-B3bwt was associated exclusively with the NE and the PM. Intranuclear expression was confined to small dots and to narrow lines that were in contact with the NE (Figure 6C, arrows). These structures probably represent canaliculi that transverse the nucleus and cytoplasmic invaginations that are lined by NE membranes and that, consequently, are decorated by the GFP chimeras (Fricker et al., 1997; Broers et al., 1999).

The GFP-B3 chimeras in which the NLS was inactivated by mutagenesis gave rise to four different staining patterns. GFP-B3awt $\Delta$ NLS was associated with endomembranes. It was also found in the nucleoplasm. No association with the NE or the PM was detected (Figure 6G). The constructs GFP-B3aCC $\Delta$ NLS and GFP-B3b6Q $\Delta$ NLS, which both contain an extra cysteine residue, showed identical staining patterns. They are associated with the PM and with the Golgi apparatus. No intranuclear or nuclear rim staining was observed (Figure 6, H and J). In contrast, GFPB3bwt $\Delta$ NLS and GFP-B3bSC $\Delta$ NLS, which both contain a basic cluster, showed nuclear rim and PM staining (Figure 6, I and K). A minor fraction of GFP-B3bSC $\Delta$ NLS was nucleoplasmic (Figure 6K). The basic cluster that is present in lamin $\mathrm{B} 3 \mathrm{~b}$ constructs has a dual function. It acts as a secondary membrane-targeting motif (Figure 6, E and K) and as a NLS. The latter was especially evident in the case of GFP$\mathrm{B} 3 \mathrm{bCS} \Delta \mathrm{NLS}$. This construct could not be farnesylated because of the mutation of the CaaX cysteine. As expected, it was not associated with membranes. Despite the inactivation of the NLS, GFP-B3bSC $\Delta$ NLS was efficiently accumulated within the nucleus (Figure 6L). Its expression pattern was indistinguishable from that of the corresponding construct that contains a functional NLS within the $\mathrm{NH}_{2}$-terminal region of the lamin B3 tail (Figure 6, compare $\mathrm{F}$ and $\mathrm{L}$ ). Furthermore, GFP-B3b6Q $\Delta$ NLS, in which the basic cluster is replaced by six glutamine residues, was exclusively cytoplasmic (Figure 6J).

The transfection experiments described above are in line with and extend recent observations on endomembrane trafficking of Ras (Choy et al., 1999). For Ras, it has been shown that the CaaX motif and a secondary targeting motif are required for PM association. Our results demonstrate that 


\section{EGFP $\triangle N L S \rightleftharpoons C a a X$}
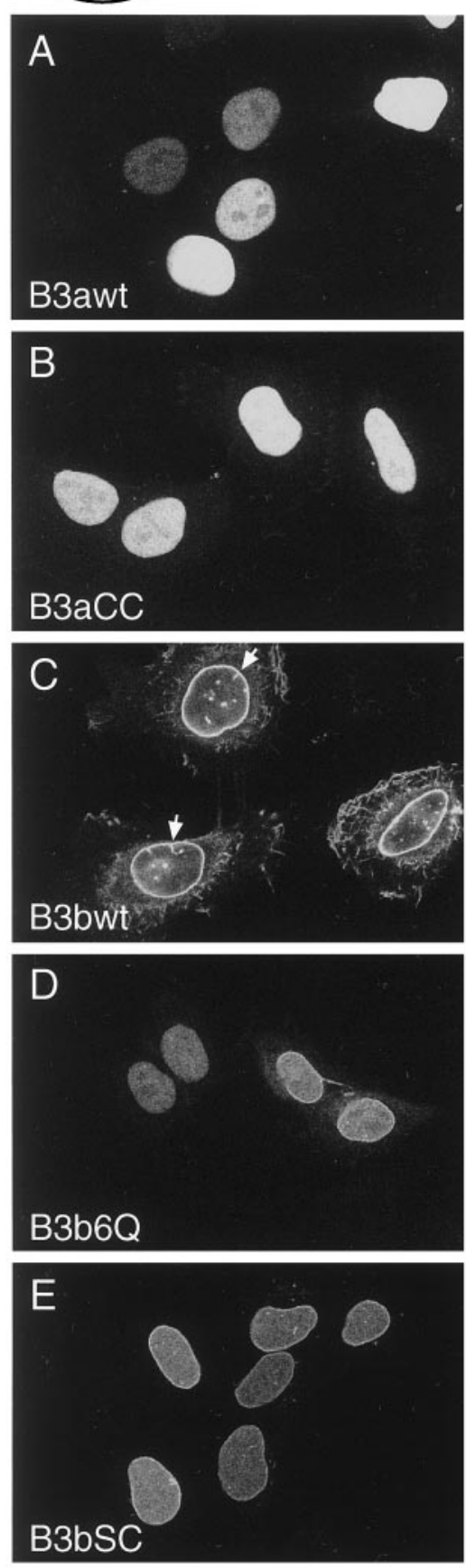

Figure 6. Differential localization of GFP lamin B3 tail chimeras. HeLa cells transiently transfected with GFP lamin tail chimeras were fixed $24 \mathrm{~h}$ after transfection and examined by fluorescence confocal laser scan microscopy. Schemes of the chimeras are given at the top. The nomenclature of the chimeras corresponds to that of the lamin constructs listed in Table 1 . The basic cluster and the palmitoylation site both contribute to NE targeting (C-E, I, and K). Targeting to the NE is most efficient in the presence of both motifs (C and I). The basic cluster acts as a NLS (I, K, and L). Constructs that lack a NLS but contain a palmitoylation site and a CaaX motif are associated with the PM, the Golgi, and peri-Golgi vesicles $(\mathrm{H}$ and J). Arrows in $\mathrm{C}$ indicate NE indentations decorated by the GFP-B3bwt chimera. Bar in L, $50 \mu \mathrm{m}$.

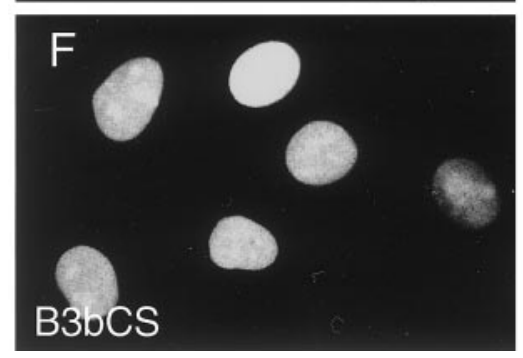

\section{B3awt $\Delta$ NLS}
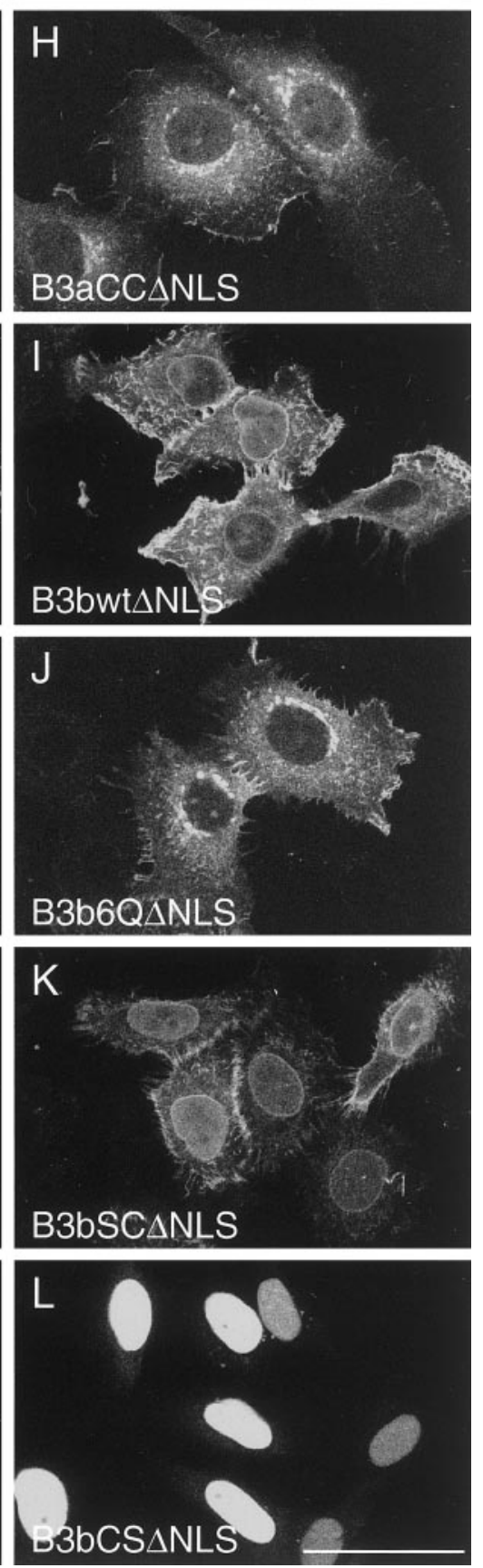
the two-signal model holds also for the association with the inner nuclear membrane. GFP chimeras that contain a secondary membrane-targeting motif in conjunction with at least one NLS showed a dual localization at the inner nuclear membrane and the PM (Figure 6, C, D, E, I, and K). Notably, GFP-B3bwt, which contains the NLS of the lamin B3 tail and a basic cluster, shows the strongest NE staining. Furthermore, nuclear rim staining was observed only with constructs that contain a NLS. This indicates that nuclear rim staining is due to expression of GFP chimeras at the inner rather than the outer nuclear membrane (Figure 6, compare $\mathrm{J}$ and $\mathrm{K})$.

To exclude the possibility that other sequences of the lamin tail than the NLS and the COOH-terminal membranetargeting motifs might influence targeting to the inner nuclear membrane, GFP chimeras were generated containing targeting sequences that were not derived from lamins. One of these chimeras contained at its $\mathrm{NH}_{2}$ terminus the NLS of the SV40 large $\mathrm{T}$ antigen followed by six myc epitopes as tags and at its $\mathrm{COOH}$ terminus the last 11 amino acid residues of human N-Ras (NLS-MT-GFP-N-Ras; for sequence, see Table 1). The N-Ras sequence is sufficient to target GFP to the PM (Choy et al., 1999). The NLS-MT-GFP$\mathrm{N}$-Ras construct was enriched at the nuclear envelope and was also located in the nucleoplasm in transfected cells (Figure 7A). The distribution between the NE and the nucleoplasm was comparable to that of the GFP lamin chimeras GFP-B3b6Q and GFP-B3bSC (Figure 6, D and E). In cells expressing the chimera at high levels, the NE showed deep invaginations that were decorated by the chimeric GFP (Figure 7B). Bright fluorescent dots were often found in the cytoplasm of transfected cells, probably resulting from the high expression. Staining of the PM was seen only in rare cases. As a control, a chimera was expressed that lacked the $\mathrm{N}$-Ras-specific sequences (NLS-MT-GFP). This protein was efficiently accumulated in the nucleoplasm. However, it showed no association with membranes (Figure 7C). In summary, these results confirm the conclusion that dual lipidation in conjunction with a NLS is sufficient to target a protein to the inner nuclear membrane.

\section{DISCUSSION}

By expressing epitope-tagged lamin B3 variants in Xenopus oocytes, we show that Xenopus lamin B3b, one of two B3 proteins that are generated by alternative RNA splicing, remains stably associated with membranes after meiotic NE breakdown, whereas lamin B3a becomes freely soluble. From previous work, it is known that the majority of lamin B3 is soluble in Xenopus eggs, whereas only a minor fraction remains associated with egg membranes (Benavente et al., 1985; Firmbach-Kraft and Stick, 1993; Lourim and Krohne, 1993; Lourim et al., 1996). Immunoblotting of two-dimensional gels allowed us to detect both protein variants in oocyte nuclei. The assignment of the multiple isoelectric variants as either lamin B3a or B3b was accomplished by expressing epitope-tagged versions of B3a or B3b in oocytes. The tagged lamins were used as markers. Immunoblots revealed that variant $\mathrm{B} 3 \mathrm{~b}$ represents a minor fraction of lamin B3 in oocytes (Figure 2A). This fits well with the relative abundance of the mRNAs encoding $\mathrm{B} 3 \mathrm{a}$ and $\mathrm{B} 3 \mathrm{~b}$ (Döring and Stick, 1990).
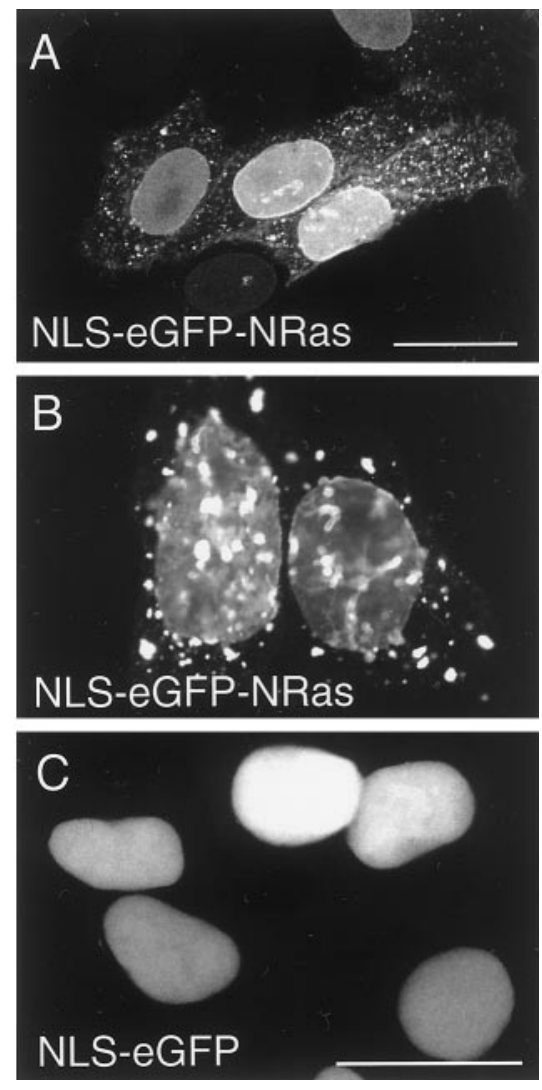

Figure 7. Minimal targeting motifs for association with the NE. HeLa cells transiently transfected with NLS-MT-GFP-N-Ras (A and B) or NLS-MT-GFP (C) chimeras were fixed $24 \mathrm{~h}$ after transfection and examined by fluorescence confocal laser scan microscopy. For sequences, see Table 1. GFP that contains a NLS at its $\mathrm{NH}_{2}$ terminus and the CaaX motif and the palmitoylation site of N-Ras at its $\mathrm{COOH}$ terminus is targeted to the NE (A and $\mathrm{B}$ ), whereas NLS-GFP without the $\mathrm{COOH}$-terminal membrane-targeting motifs is accumulated within the nucleoplasm (C). Note that cells expressing NLSGFP-N-Ras show deep invaginations of the NE that are decorated by the chimeric GFP. Cells in B and $C$ are shown at the same magnification. Bars in A and C, $25 \mu \mathrm{m}$.

The presence of soluble and membrane-bound lamin B3 in the amphibian egg cytoplasm, together with earlier observations that soluble lamin B3 retained its CaaX motif-mediated modifications (isoprenylation and carboxyl methylation) in mature eggs, led us to conclude that these modifications, although necessary, are not sufficient for stable association of lamins with NE-derived membranes (Firmbach-Kraft and Stick, 1993). The existence of two lamin B3 variants in oocytes that differ in their membrane-binding properties can explain the earlier observations at the molecular level.

Our RT-PCR data show that the relative abundance of the mRNAs of the two variants is developmentally regulated (Figure 1). This is also reflected at the protein level. Variant $\mathrm{B} 3 \mathrm{~b}$ is a minor species in oocytes (this study) but is much more abundant relative to variant $\mathrm{B} 3 \mathrm{a}$ in later stages of development (see Figure 5d in Stick and Hausen, 1985). The 
biological relevance of the existence of a soluble and a membrane-bound pool of the same lamin and the developmental regulation of the relative proportions of these pools is not clear at present (Fricker et al., 1997; Gant and Wilson, 1997; Lourim and Krohne, 1998).

Both lamin variants contain a CaaX motif, and both are isoprenylated. However, lamin B3b contains, in addition, a basic cluster and a potential palmitoylation site adjacent to the CaaX motif. It has been shown that palmitoylation or a basic cluster in conjunction with $\mathrm{CaaX}$-dependent modifications confer stable association of Ras proteins with the PM (Hancock et al., 1990). The concepts of double lipidation (isoprenylation plus S-acylation) and the two-signal mechanism (lipidation plus basic cluster) for stable membrane association also applies to other groups of lipid-modified proteins, such as those subject to $\mathrm{NH}_{2}$-terminal myristoylation (Cadwallader et al., 1994; Resh, 1996). All of these proteins localize at the PM or to the Golgi. Our results show that the same principles also hold for proteins that bind to the inner NE membrane. This became particularly clear from the results obtained with mutant B3 variants. Replacement of the serine residue with cysteine next to the CaaX results in stable association of B3a with NE membranes in the Xenopus oocyte/egg system (Figure 4). Conversely, changing the palmitoyl cysteine of lamin B3b to a serine has the opposite effect. It grossly reduces membrane binding, although binding of B3bSC is not completely abolished. This indicates that lamin B3b, like $\mathrm{H}$ - and N-Ras, is stably associated with membranes via double lipidation by an isoprene plus a palmitoyl residue. The basic cluster that is present in lamin $\mathrm{B} 3 \mathrm{~b}$ adds to membrane binding, although its contribution is relatively small in the oocyte/egg system. Both the effect of palmitoylation and the basic cluster depend on the CaaX modifications, as revealed by a mutant in which the CaaX cysteine had been replaced by serine.

We were unable to demonstrate directly the presence of a palmitoyl residue on lamin B3b. This is due to technical reasons. The small amount of endogenous B3b in oocytes precluded direct chemical identification of a palmitoylcysteine residue in $\mathrm{B} 3 \mathrm{~b}$. In vivo labeling of $\mathrm{B} 3 \mathrm{~b}$ with radiolabeled palmitate was also unsuccessful. This is probably due to the reversible nature of palmitoylation and the fact that palmitate is rapidly metabolized in cells (Kloc et al., 1991; Casey and Buss, 1995). In contrast to tissue culture cells, in amphibian oocytes incorporation of newly synthesized lamins into the NE is a relatively slow process, making radiolabeling with palmitate extremely inefficient. Nevertheless, the label consistently was three to five times greater in immunoisolates of $\left[{ }^{3} \mathrm{H}\right]$ palmitate-labeled oocytes that had been injected with RNA encoding lamin B3b than in oocytes injected with B3a RNA.

Geranylgeranyl-modified Ras has been shown to bind more tightly to membranes than farnesylated Ras (Hancock et al., 1991b). Determination of the affinities of farnesylated versus geranylgeranylated model peptides to lipid vesicles shows a higher affinity of the latter (Silvius and l'Heureux, 1994). Moreover, in conjunction with a basic cluster, geranylgeranylation is sufficient to target a protein to the PM in tissue culture cells (Hancock et al., 1991b). Geranylgeranylated lamin B3a, like the farnesylated wild-type protein, became soluble after meiotic NE breakdown (Figure 5). Obviously, an increase in hydrophobicity alone is not sufficient for stable association with NE-derived membranes. In light of the two-signal mechanism proposed for Ras PM binding, it would be interesting to determine whether the introduction of a basic cluster into B3aCAIL would result in membrane association. Such experiments have not yet been done.

The transfection experiments carried out with chimeras of GFP and the $\mathrm{COOH}$ regions of wild-type and mutant lamin B3b demonstrate that the same signals that confer association with nuclear membranes in the amphibian oocyte/egg system are sufficient to target a heterologous protein to the inner nuclear membrane in human tissue culture cells. Even more strikingly, several of the chimeras that contain at least one functional NLS showed a dual location at the NE and the PM. Lamins are never found in association with cytoplasmic membranes during interphase. The dual location of the chimeras in the transfected cells is probably due to overexpression. However, it suggests similarities between the PM and the inner nuclear membrane with respect to binding of lipidated proteins.

The basic cluster of lamin B3b exerts a dual function. It acts as a NLS and as a secondary membrane-targeting signal. Its NLS function is especially clear in construct GFPB3bCS $\Delta$ NLS (Figure 6L). This construct cannot be modified by lipidation and shows no affinity to membranes. It is accumulated in nuclei with the same efficiency as the corresponding construct that carries the wild-type lamin NLS. A similar property was described for the polybasic cluster of K-Ras (Hancock et al., 1990).

The CaaX motif alone is sufficient to target a protein to the endomembrane system, but it is insufficient for PM targeting (Choy et al., 1999). Similar to our observations in the amphibian oocyte/egg system, it is also insufficient on its own to confer stable binding to the NE. This observation is somewhat at variance with previous findings (Hennekes and Nigg, 1994) that showed at least week nuclear rim staining of chimeras of pyruvate kinase and chicken lamin tails, a phenotype we did not observe with the GFP-B3awt construct (Figure 6A).

Our results show that both the basic cluster and the palmitoylation site contribute to NE targeting in the transfected cells. Chimeras that lack either of the two signals show nuclear as well as nucleoplasmic staining (Figure 6, D, E, and $\mathrm{K}$ ), whereas the construct that contains both signals is located exclusively at the NE and the PM (Figure 6C).

Unexpectedly, GFP-B3aCC is not targeted to the NE, whereas the corresponding chimera lacking the NLS is associated with the Golgi and the PM (Figure 6, B and H). Although we do not have a straightforward explanation, it might be that, as observed in other cases (El-Husseini et al., 2000), palmitoylation is influenced by amino acid residues surrounding the palmitoyl cysteine. This would imply that different palmitoyltransferases are active in the cytoplasm and the nucleus (see below) and that these enzymes might differ in their optimal sequence requirements.

The striking similarity between the targeting signals of Ras and those found in nuclear lamin B3b of Xenopus, together with our finding that these signals can target proteins to both cytoplasmic and nuclear membrane compartments, raises the question of how trafficking to the two destinations occurs. For Ras, a detailed description was given recently (Choy et al., 1999). Ras proteins are made in the cytoplasm and are farnesylated by soluble 
farnesyltransferase (Casey and Seabra, 1996). They then bind to the ER and are aaX proteolyzed and carboxyl methylated. The aaX protease and the pcCMT are integral membrane proteins of the ER (Magee and Marshall, 1999). $\mathrm{H}-\mathrm{R}$ as and N-Ras then traffic to the PM via the secretory pathway, whereas K-Ras is probably targeted directly to the PM (Yokoe and Meyer, 1996; Choy et al., 1999; Apolloni et al., 2000). The association of K-Ras with the PM is assisted by its basic cluster. S-Acylation of $\mathrm{H}$ - and N-Ras occurs in an early compartment of the exocytotic pathway (Choy et al., 1999; Magee and Marshall, 1999; Apolloni et al., 2000). Studies with lipid-modified peptides have shown that efficient S-acylation can occur directly at the PM (Schroeder et al., 1996) and that doubly lipidated peptides are bound to membrane vesicles by kinetic trapping (Shahinian and Silvius, 1995). Whether K-Ras exists in a dynamic equilibrium and can rapidly switch between a PM-bound and a cytosolic form (Yokoe and Meyer, 1996) or shows fast lateral diffusion at the PM (Niv et al., 1999) is still a matter of debate. Our results with mutant lamin B3 in the amphibian system indicate that palmitoylation strongly contributes to binding to the inner nuclear membrane. Therefore, lamin B3b should be trapped as soon as palmitoylation has occurred. We know from earlier studies that farnesylation of lamin B3 occurs in the cytoplasm before its uptake into the nucleus (FirmbachKraft and Stick, 1995). The intriguing question remains at which point the transport pathways of doubly lipidated proteins destined to either the PM or the inner NE will deviate. Lamin B3b might be palmitoylated after nuclear transport by a palmitoyltransferase located at the inner nuclear membrane. Alternatively, it might be doubly lipidated at cytoplasmic membranes and targeted to the inner nuclear membrane with the aid of escort protein(s) that are able to extract the doubly lipidated lamins from the cytoplasmic membranes. Injection experiments with enucleated oocytes showed that neither of the two Flag-B3 variants associated with endomembranes. Molecular characterization of the S-acylation machinery and its precise subcellular localization (Dunphy and Linder, 1998; Apolloni et al., 2000; Reuther and Der, 2000) might help decide which of these two possibilities is correct.

Whether the same mechanism described here for an amphibian lamin also holds for other lamins is not clear. B-type lamins of mammals and birds are associated with mitotic NE-derived membranes (Gerace and Blobel, 1980; Stick et al., 1988; Meier and Georgatos, 1994). The cDNA sequences of the two avian and several mammalian B-type lamins indicate that all of these proteins carry a CaaX motif, but none contains additional membrane-targeting signals such as a basic cluster or an extra cysteine residue. Stable membrane association of these lamins during mitosis must be mediated by other factors. Several integral membrane proteins of the inner nuclear membrane have been shown to interact with lamins in vitro and in vivo. Whether these act as bona fide lamin membrane receptors, however, is still a matter of debate (Gant and Wilson, 1997; Gotzmann and Foisner, 1999).

\section{ACKNOWLEDGMENTS}

We thank T. Pieler, Göttingen, for his generous support. We are grateful to M. Philips, New York, for providing us with GFP-Ras constructs and Katja Köbernik, Göttingen, for the Flag version of the pCS vector. We thank H. Spring, Heidelberg, for help with the laser scan microscopy and T. Ralle, Heidelberg, for his contribution to the cloning of the GFP-N-Ras construct. We thank Caroline Keedy, Göttingen, and D. Olins, Scarborough, for critically reading the manuscript. The initial phase of this work was carried out during our stay at the Institut für Biochemie, Universität Göttingen, and was supported by the Deutsche Forschungsgemeinschaft (SFB 523 A3).

\section{REFERENCES}

Apolloni, A., Prior, I.A., Lindsay, M., Parton, R.G., and Hancock, J.F. (2000). H-ras but not K-ras traffics to the plasma membrane through the exocytic pathway. Mol. Cell. Biol. 20, 2475-2487.

Beck, L.A., Hosick, T.J., and Sinensky, M. (1990). Isoprenylation is required for the processing of the lamin A precursor. J. Cell Biol. 110, 1489-1499.

Benavente, R., Krohne, G., and Franke, W.W. (1985). Cell typespecific expression of nuclear lamina proteins during development of Xenopus laevis. Cell 41, 177-190.

Broers, J.L., Machiels, B.M., van Eys, G.J., Kuijpers, H.J., Manders, E.M., van Driel, R., and Ramaekers, F.C. (1999). Dynamics of the nuclear lamina as monitored by GFP-tagged A-type lamins. J. Cell Sci. 112, 3463-3475.

Cadwallader, K.A., Paterson, H., Macdonald, S.G., and Hancock, J.F. (1994). N-terminally myristoylated Ras proteins require palmitoylation or a polybasic domain for plasma membrane localization. Mol. Cell. Biol. 14, 4722-4730.

Casey, P.H., and Buss, J.E. (1995). Lipid modifications of proteins. Methods Enzymol. 250, 314-336.

Casey, P.J., and Seabra, M.C. (1996). Protein prenyltransferases. J. Biol. Chem. 271, 5289-5292.

Choy, E., Chiu, V.K., Silletti, J., Feoktistov, M., Morimoto, T., Michaelson, D., Ivanov, I.E., and Philips, M.R. (1999). Endomembrane trafficking of Ras: the CAAX motif targets proteins to the ER and Golgi. Cell 98, 69-80.

Dai, Q., Choy, E., Chiu, V., Romano, J., Slivka, S.R., Steitz, S.A., Michaelis, S., and Philips, M.R. (1998). Mammalian prenylcysteine carboxyl methyltransferase is in the endoplasmic reticulum. J. Biol. Chem. 273, 15030-15034.

Döring, V., and Stick, R. (1990). Gene structure of nuclear lamin LIII of Xenopus laevis: a model for the evolution of IF proteins from a lamin-like ancestor. EMBO J. 9, 4073-4081.

Dudler, T., and Gelb, M.H. (1996). Palmitoylation of Ha-Ras facilitates membrane binding, activation of downstream effectors, and meiotic maturation in Xenopus oocytes. J. Biol. Chem. 271, 1154111547.

Dunphy, J.T., and Linder, M.E. (1998). Signaling functions of protein palmitoylation. Biochim. Biophys. Acta 1436, 245-261.

El-Husseini, A.E., Craven, S.E., Chetkovich, D.M., Firestein, B.L., Schnell, E., Aoki, C., and Bredt, D.S. (2000). Dual palmitoylation of PSD-95 mediates its vesiculotubular sorting, postsynaptic targeting, and ion channel clustering. J. Cell Biol. 148, 159-172.

Firmbach-Kraft, I., and Stick, R. (1993). The role of CaaX-dependent modifications in membrane association of Xenopus nuclear lamin B3 during meiosis and the fate of B3 in transfected mitotic cells. J. Cell Biol. 123, 1661-1670.

Firmbach-Kraft, I., and Stick, R. (1995). Analysis of nuclear lamin isoprenylation in Xenopus oocytes: isoprenylation of lamin B3 precedes its uptake into the nucleus. J. Cell Biol. 129, 17-24. 
Fricker, M., Hollinshead, M., White, N., and Vaux, D. (1997). Interphase nuclei of many mammalian cell types contain deep, dynamic, tubular membrane-bound invaginations of the nuclear envelope. J. Cell Biol. 136, 531-544.

Gant, T.M., and Wilson, K.L. (1997). Nuclear assembly. Annu. Rev. Cell Dev. Biol. 13, 669-695.

Gerace, L., and Blobel, G. (1980). The nuclear envelope lamina is reversibly depolymerized during mitosis. Cell 19, 277-287.

Glomset, J.A., and Farnsworth, C.C. (1994). Role of protein modification reactions in programming interactions between ras-related GTPases and cell membranes. Annu. Rev. Cell Biol. 10, 181-205.

Gotzmann, J., and Foisner, R. (1999). Lamins and lamin-binding proteins in functional chromatin organization. Crit. Rev. Eukaryot. Gene Expr. 9, 257-265.

Hancock, J.F., Cadwallader, K., and Marshall, C.J. (1991a). Methylation and proteolysis are essential for efficient membrane binding of prenylated p21K-ras(B). EMBO J. 10, 641-646.

Hancock, J.F., Cadwallader, K., Paterson, H., and Marshall, C.J. (1991b). A CAAX or a CAAL motif and a second signal are sufficient for plasma membrane targeting of ras proteins. EMBO J. 10, 40334039 .

Hancock, J.F., Magee, A.I., Childs, J.E., and Marshall, C.J. (1989). All ras proteins are polyisoprenylated but only some are palmitoylated. Cell 57, 1167-1177.

Hancock, J.F., Paterson, H., and Marshall, C.J. (1990). A polybasic domain or palmitoylation is required in addition to the CAAX motif to localize p21ras to the plasma membrane. Cell 63, 133-139.

Hennekes, H., and Nigg, E.A. (1994). The role of isoprenylation in membrane attachment of nuclear lamins: a single point mutation prevents proteolytic cleavage of the lamin A precursor and confers membrane binding properties. J. Cell Sci. 107, 1019-1029.

Holtz, D., Tanaka, R.A., Hartwig, J., and McKeon, F. (1989). The CaaX motif of lamin A functions in conjunction with the nuclear localization signal to target assembly to the nuclear envelope. Cell 59, 969-977.

Huttenlauch, I., Peck, R.K., and Stick, R. (1998). Articulins and epiplasmins: two distinct classes of cytoskeletal proteins of the membrane skeleton in protists. J. Cell Sci. 111, 3367-3378.

Jackson, J.H., Li, J.W., Buss, J.E., Der, C.J., and Cochrane, C.G. (1994). Polylysine domain of K-ras $4 \mathrm{~B}$ protein is crucial for malignant transformation. Proc. Natl. Acad. Sci. USA 91, 12730-12734.

Kilic, F., Johnson, D.A., and Sinensky, M. (1999). Subcellular localization and partial purification of prelamin A endoprotease: an enzyme which catalyzes the conversion of farnesylated prelamin A to mature lamin A. FEBS Lett. 450, 61-65.

Kitten, G.T., and Nigg, E.A. (1991). The CaaX motif is required for isoprenylation, carboxyl methylation, and nuclear membrane association of lamin B2. J. Cell Biol. 113, 13-23.

Kloc, M., Reddy, B., Crawford, S., and Etkin, L.D. (1991). A novel $110-\mathrm{kDa}$ maternal CAAX box-containing protein from Xenopus is palmitoylated and isoprenylated when expressed in baculovirus. J. Biol. Chem. 266, 8206-8212.

Krohne, G., Waizenegger, I., and Höger, T.H. (1989). The conserved carboxy-terminal cysteine of nuclear lamins is essential for lamin association with the nuclear envelope. J. Cell Biol. 109, 2003-2011.

Loewinger, L., and McKeon, F. (1988). Mutations in the nuclear lamin proteins resulting in their aberrant assembly in the cytoplasm. EMBO J. 7, 2301-2309.

Lourim, D., Kempf, A., and Krohne, G. (1996). Characterization and quantitation of three B-type lamins in Xenopus oocytes and eggs: increase of lamin LI protein synthesis during meiotic maturation. J. Cell Sci. 109, 1775-1785.

Lourim, D., and Krohne, G. (1993). Membrane-associated lamins in Xenopus egg extracts: identification of two vesicle populations. J. Cell Biol. 123, 501-512.

Lourim, D., and Krohne, G. (1998). Chromatin binding and polymerization of the endogenous Xenopus egg lamins: the opposing effects of glycogen and ATP. J. Cell Sci. 111, 3675-3686.

Magee, T., and Marshall, C. (1999). New insights into the interaction of Ras with the plasma membrane. Cell 98, 9-12.

Matthews, G., and Colman, A. (1991). A highly efficient, cell-free translation/translocation system prepared from Xenopus eggs. Nucleic Acids Res. 19, 6405-6412.

Meier, J., and Georgatos, S.D. (1994). Type B lamins remain associated with the integral nuclear envelope protein p58 during mitosis: implications for nuclear reassembly. EMBO J. 13, 1888-1898.

Moores, S.L., Schaber, M.D., Mosser, S.D., Rands, E., O'Hara, M.B., Garsky, V.M., Marshall, M.S., Pompliano, D.L., and Gibbs, J.B. (1991). Sequence dependence of protein isoprenylation. J. Biol. Chem. 266, 14603-14610.

Nigg, E.A., Kitten, G.T., and Vorburger, K. (1992). Targeting lamin proteins to the nuclear envelope: the role of CaaX box modifications. Biochem. Soc. Trans. 20, 500-504.

Niv, H., Gutman, O., Henis, Y.I., and Kloog, Y. (1999). Membrane interactions of a constitutively active GFP-Ki-Ras 4B and their role in signaling: evidence from lateral mobility studies. J. Biol. Chem. 274, 1606-1613.

Ottaviano, Y., and Gerace, L. (1985). Phosphorylation of the nuclear lamins during interphase and mitosis. J. Biol. Chem. 260, 624-632.

Reiss, Y., Stradley, S.J., Gierasch, L.M., Brown, M.S., and Goldstein, J.L. (1991). Sequence requirement for peptide recognition by rat brain p21ras protein farnesyltransferase. Proc. Natl. Acad. Sci. USA 88, 732-736.

Resh, M.D. (1996). Regulation of cellular signaling by fatty acid acylation and prenylation of signal transduction proteins. Cell Signal. $8,403-412$.

Reuther, G.W., and Der, C.J. (2000). The Ras branch of small GTPases: Ras family members don't fall far from the tree. Curr. Opin. Cell Biol. 12, 157-165.

Rupp, R.A., Snider, L., and Weintraub, H. (1994). Xenopus embryos regulate the nuclear localization of XMyoD. Genes Dev. 8, 13111323.

Schafer, W.R., and Rine, J. (1992). Protein prenylation: genes, enzymes, targets, and functions. Annu. Rev. Genet. 26, 209-237.

Schroeder, H., Leventis, R., Shahinian, S., Walton, P.A., and Silvius, J.R. (1996). Lipid-modified, cysteinyl-containing peptides of diverse structures are efficiently S-acylated at the plasma membrane of mammalian cells. J. Cell Biol. 134, 647-660.

Shahinian, S., and Silvius, J.R. (1995). Doubly-lipid-modified protein sequence motifs exhibit long-lived anchorage to lipid bilayer membranes. Biochemistry. 34, 3813-3822.

Silvius, J.R., and l'Heureux, F. (1994). Fluorimetric evaluation of the affinities of isoprenylated peptides for lipid bilayers. Biochemistry 33, 3014-3022.

Stick, R. (1987). Dynamics of the nuclear lamina during mitosis and meiosis. In: Molecular Regulation of Nuclear Events in Mitosis and Meiosis, ed. R.A. Schlegel, M.S. Hallek, and P.N. Rao, New York: Academic Press, 43-66.

Stick, R. (1988). cDNA cloning of the developmentally regulated lamin LIII of Xenopus laevis. EMBO J. 7, 3189-3197. 
Stick, R., Angres, B., Lehner, C.F., and Nigg, E.A. (1988). The fates of chicken nuclear lamin proteins during mitosis: evidence for a reversible redistribution of lamin $\mathrm{B} 2$ between inner nuclear membrane and elements of the endoplasmic reticulum. J. Cell Biol. 107, 397406.

Stick, R., and Hausen, P. (1985). Changes in the nuclear lamina composition during early development of Xenopus laevis. Cell 41, 191-200.

Stick, R., and Krohne, G. (1982). Immunological localization of the major architectural protein associated with the nuclear envelope of the Xenopus laevis oocyte. Exp. Cell Res. 138, 319-323.

Weber, K., Plessmann, U., and Traub, P. (1989). Maturation of nuclear lamin A involves a specific carboxy-terminal trimming, which removes the polyisoprenylation site from the precursor: implications for the structure of the nuclear lamina. FEBS Lett. 257, 411-414.
Wessel, D., and Flügge, U.I. (1984). A method for the quantitative recovery of protein in dilute solution in the presence of detergents and lipids. Anal. Biochem. 138, 141-143.

Willumsen, B.M., Cox, A.D., Solski, P.A., Der, C.J., and Buss, J.E. (1996). Novel determinants of H-Ras plasma membrane localization and transformation. Oncogene 13, 1901-1909.

Wilson, K.L., and Newport, J. (1988). A trypsin-sensitive receptor on membrane vesicles is required for nuclear envelope formation in vitro. J. Cell Biol. 107, 57-68.

Yokoe, H., and Meyer, T. (1996). Spatial dynamics of GFP-tagged proteins investigated by local fluorescence enhancement. Nat. Biotechnol. 14, 1252-1256.

Zhang, F.L., and Casey, P.J. (1996). Protein prenylation: molecular mechanisms and functional consequences. Annu. Rev. Biochem. 65, 241-269. 